\title{
The prognostic effects of tumor infiltrating regulatory $T$ cells and myeloid derived suppressor cells assessed by multicolor flow cytometry in gastric cancer patients
}

\author{
Han Sol Choi ${ }^{1,2, *}$, Sang Yun Ha ${ }^{3, *}$, Hye-Mi Kim ${ }^{1,2}$, Soo Min Ahn ${ }^{3}$, Myung-Soo Kang ${ }^{4,5}$, \\ Kyoung-Mee Kim ${ }^{3}$, Min Gew Choi ${ }^{6}$, Joon Ho Lee ${ }^{6}$, Tae Sung Sohn' ${ }^{6}$ Jae Moon Bae ${ }^{6}$, \\ Sung Kim ${ }^{6}$, Eun-Suk Kang ${ }^{2}$ \\ ${ }^{1}$ Samsung Biomedical Research Institute, Samsung Medical Center, Sungkyunkwan University School of Medicine, Seoul, Korea \\ ${ }^{2}$ Department of Laboratory Medicine and Genetics, Samsung Medical Center, Sungkyunkwan University School of Medicine, \\ Seoul, Korea \\ ${ }^{3}$ Department of Pathology and Translational Genomics, Samsung Medical Center, Sungkyunkwan University School of \\ Medicine, Seoul, Korea \\ ${ }^{4}$ Samsung Advanced Institute for Health Sciences and Technology (SAIHST), Samsung Medical Center, Sungkyunkwan \\ University School of Medicine, Seoul, Korea \\ ${ }^{5}$ Samsung Biomedical Research Institute (SBRI), Center for Future Sciences, Samsung Medical Center, Sungkyunkwan \\ University School of Medicine, Seoul, Korea \\ ${ }^{6}$ Department of Surgery, Samsung Medical Center, Sungkyunkwan University School of Medicine, Seoul, Korea \\ "These author contributed equally to this work
}

Correspondence to: Eun-Suk Kang, e-mail: eskang@skku.edu Kyoung-Mee Kim, e-mail: kkmkys@skku.edu

Keywords: regulatory $T$ cells, myeloid derived suppressor cells, prognosis, gastric cancer

Received: April 17, 2015

Accepted: December 26, 2015

Published: January 20, 2016

\section{ABSTRACT}

The prognostic effects of tumor infiltrating lymphocytes (TILs), especially regulatory $\mathbf{T}$ cells (Tregs) and myeloid derived suppressing cells (MDSCs) are inconclusive in gastric cancers. We investigated the frequencies of TILs including CD8+ T cells, CD45+CD4+CD25 F FXP3+ Tregs, CD45+CD11b+ CD14+ HLA-DR- MDSCs in 28 gastric cancer tissues by using multicolor flow cytometry. In gastric cancer tissue, the percentage of Tregs among the CD4+ $T$ cell subset was substantially increased compared to that of Tregs among peripheral blood CD4+ $T$ cells from the controls. High frequency of $\mathrm{CD} 8+\mathrm{T}$ cells among $\mathrm{CD} 3+\mathrm{T}$ cells correlated with increased overall survival $(O S)(p=0.005)$. High frequency of Tregs among CD4+ T cells correlated with increased OS $(p<0.001)$, and disease-free survival (DFS) $(p=0.039)$ and was an independent prognostic factor in OS (Hazard ratio: $0.047 ; 95 \%$ confidence interval, 0.006-0.372; $p=0.004)$. High frequency of MDSCs among total examined cells correlated with decreased OS $(p=0.027)$ and was an independent prognostic factor in OS (Hazard ratio 8.601; 95\% confidence interval, 1.240-59.678; $p=0.029$ ). We have demonstrated that high levels of Tregs among tumor-infiltrating CD4+ $T$ cells were favorable, but an increased proportion of MDSCs was an adverse independent prognostic factor in gastric cancer. Our results may provide important insights for future immunotherapy in gastric cancer.

\section{INTRODUCTION}

Gastric cancer (GC) is the fourth most commonly diagnosed cancer and the second most common cause of cancer-related deaths worldwide $[1,2]$. Despite efforts to introduce new treatment modalities such as surgery combined with chemotherapy or chemo-radiotherapy, the control of GC at an advanced stage remains difficult [3]. Considering the high mortality rate of GC in advanced stage or at recurrence, new therapeutic strategies are urgently needed.

Mechanisms underlying the dynamic interplay between immune cells and tumor progression have 
been studied during past decades. The accumulated data indicate that the outcome of an immune response toward a tumor is largely determined by the type of immune response elicited [4]. Earlier studies have shown survival benefit of tumor infiltrating lymphocytes (TILs) [5-8], and this suggests that TILs are effective on suppressing tumor progression. Thereafter, subgrouping of CD3+ $\mathrm{T}$ cells into cytotoxic $\mathrm{T}$ cells and regulatory $\mathrm{T}$ cells (Tregs) have made further understanding of relationship between TILs and cancer progression. Infiltration of CD8+ cytotoxic T cells into tumors has been reported as a favorable prognostic factor in many cancers including GCs [9-17]. Tregs regulate immune reactivity including tumor-specific immune responses [18]. Tregs were initially characterized by the CD4+CD25+ phenotype, but the most specific cell marker of Tregs identified to date is the nuclear transcription factor known as Forkhead box protein P3 (FOXP3) [19-23]. Many studies have shown that a high density of Tregs, which usually evaluated by quantification of FOXP3 + T cells by using immunohistochemistry (IHC), was associated with a poor outcome, and these results were explained on the basis of the suppressive effects of Tregs on antitumor cytotoxic T cells [24-26]. However, many controversial results have been reported [24, 27]. Moreover, the controversial prognostic significance of Tregs may differ depending on the specific tumor types. According to a recent meta-analyses, the prognostic impacts of Treg infiltration is not consistent [27]. For example, Treg is a favorable prognostic factor in colorectal carcinoma while it is an adverse prognostic factor in hepatocellular carcinoma [24]. In GC, the prognostic significance of Tregs has been controversial. Many studies reported Tregs as adverse prognostic factor in GC [25, 27-29], while several opposite results suggesting Tregs as a favorable prognostic factor have been reported so far $[18,30,31]$.

Myeloid-derived suppressor cells (MDSCs) are a heterogeneous population of immature myeloid cells and are involved in inhibiting both innate and adaptive immune responses [32, 33]. In cancer biology, MDSCs have been shown to potently inhibit antitumor immunity in vivo through diverse mechanisms [32, 33]. MDSCs have been generally defined as a CD11b+ CD33+ CD14+ HLADR- myeloid cell population in human cancer patients [33]. In $\mathrm{GC}$, two previous studies have shown that the numbers of MDSCs are increased in the blood of cancer patients compared with healthy individuals, and this increase was associated with adverse clinical outcomes [34, 35]. However, the clinical significance of MDSCs in GC tissue remains completely unknown because specific phenotype of MDSCs cannot be evaluated by IHC in cancer tissue.

In this study, we examined the frequencies of tumor infiltrating immune cell subsets by multi-color flow cytometry, which enabled us to define more specific roles of immune cells as well as more objective quantification in $\mathrm{GC}$, and investigated the clinical significance of immune cells, especially Treg and MDSC.

\section{RESULTS}

\section{Distribution of immune cell subsets in gastric cancer tissue}

The frequencies of the various immune cell subsets in the GC tissue are summarized in Table 1. The frequencies of immune cell subsets in PBMCs of 8 healthy individuals measured using same immunophenotyping panels are displayed. In GC tissue samples, CD45+ hematopoietic cells occupied $8.5 \%$ (median value, range $0.8-29.4 \%$ ) of the total counted cells, and lymphocytes and myeloid cells occupied $68.4 \%$ and $31.6 \%$ of the CD45+ cells, respectively.

The percentage of Tregs among the CD4+ T cells and MDSCs among CD45+ leukocytes in GC tissue from cancer patients was median $12.7 \%$ (range $1.7-37.6 \%$ ) and median $2.8 \%$ (range $0.6-13.6 \%$ ), respectively. The percentage of Tregs among the CD4+ T cells and MDSCs among CD45+ leukocytes in peripheral blood from healthy donors was median $4.1 \%$ (range $0.5-8.5 \%$ ) and median $0.35 \%$ (range $0.1-3.4 \%$ ), respectively. Unexpectedly, CD56+ NK cells were scarcely observed in the GC tissues (proportion of NK cells among lymphocytes, median $0.1 \%$; range $0-3 \%$ ).

\section{The classification according to frequencies of immune cells by two strategies: among total examined cells or their belonging subset of immune cells}

The cases were dichotomized into low-density and high-density group by two strategies: (1). Frequencies among total examined cells; (2). Frequencies among their belonging subsets of immune cells. According to the frequency of CD8+ T cells (CD8/Total), Tregs (Treg/Total), and MDSCs (MDSC/Total) among total examined, cases were sub-classified into the low density group (CD8 ${ }^{\text {low/Total, Treg }}{ }^{\text {low }} /$ Total, MDSC ${ }^{\text {low}} /$ Total) and the high density groups (CD $8^{\text {high}} /$ Total, Treg ${ }^{\text {high}} /$ Total, $\mathrm{MDSC}^{\text {high}} /$ Total) based on the cutoff determined using the X-tile package (CD8/Total, 2.0\%; Treg/Total, 0.7\%; MDSC/Total, $0.24 \%$ ). According to the frequency of CD8+ T cells among CD3 + T cells (CD8/CD3), Tregs among CD4+ helper T cells (Treg/CD4), and MDSCs among CD45+ leukocytes (MDSC/CD45), cases were dichotomized into the low density $\left(\mathrm{CD} 8^{\text {low }} / \mathrm{CD} 3\right.$, Treg ${ }^{\text {low }} /$

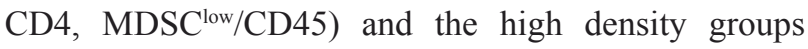
(CD8 ${ }^{\text {high }} / \mathrm{CD} 3$, Treg $\left.{ }^{\text {high} / C D 4, ~ M D S C ~}{ }^{\text {high}} / C D 45\right)$ based on the cutoff levels determined with X-tile package as follows: CD8/CD3, 30\%; Treg/CD4, 9\%; MDSC/CD45, 2.2\%. The associations of clinicopathologic features and frequencies of immune cells among total examined cells and their belonging subsets of immune cells were summarized in Table 2 and 3. 
Table 1: Distribution of lymphocyte subsets in gastric cancer tissue and peripheral blood mononuclear cells of healthy individuals

\begin{tabular}{|l|l|c|c|}
\hline \multicolumn{2}{c}{ Immunophenotypes } & \multicolumn{1}{c}{ Subsets } & $\begin{array}{c}\text { Gastric cancer tissue } \\
(\boldsymbol{n}=\mathbf{2 8})\end{array}$ \\
\cline { 3 - 5 } & \multicolumn{1}{c}{$\begin{array}{c}\text { Peripheral blood from } \\
\text { healthy control } \\
(\boldsymbol{n}=\mathbf{8})\end{array}$} \\
\hline CD45+ leukocyte among total cells & Leukocytes & $8.45(0.8-29.4)$ & 100 \\
\hline $\begin{array}{l}\text { Lymphocyte (size gating) among CD45+ } \\
\text { leukocytes }\end{array}$ & Lymphocyte & $4.15(0.3-22.0)$ & $19.3(14.4-28.4)$ \\
\hline CD3+CD4+ among CD3+ cells & Helper T cells & $56.6(20.9-83.4)$ & $59.4(29.1-74.0)$ \\
\hline CD4+FOXP3+ among CD4+ cells & Regulatory T cells & $12.7(1.7-37.6)$ & $4.1(0.5-8.5)$ \\
\hline CD3+CD8+ among CD3+ cells & Cytotoxic T cells & $42.2(16.6-79.1)$ & $38.3(3.1-47.2)$ \\
\hline CD56+ among lymphocytes & NK cells & $0.1(0-3.0)$ & $10.3(1.3-27.1)$ \\
\hline $\begin{array}{l}\text { CD45+CD11b+CD14+ HLA-DR- among } \\
\text { CD45+ leukocytes }\end{array}$ & MDSCs & $2.8(0.6-13.6)$ & $0.35(0.1-3.4)$ \\
\hline
\end{tabular}

High frequency of CD8+ $\mathrm{T}$ cells among CD3+ $T$ cells correlates with increased overall survival

The CD ${ }^{\text {high }} /$ Total patient subgroup was associated with longer DFS and OS compared with the CD8 ${ }^{\text {low }}$ Total patient subgroup, but the tendency did not reach statistical significance (Figure 1A, 1D). The proportion of the $\mathrm{CD} 8^{\text {high }} / \mathrm{CD} 3$ group was tended to increase according to the advancing $\mathrm{N}$ stage $(p=0.023)$ (Table 3$)$. The $\mathrm{CD} 8^{\text {high }} / \mathrm{CD} 3$ group showed longer OS $(p=0.005)$ and a tendency towards longer DFS than the $\mathrm{CD} 8^{\text {low }} / \mathrm{CD} 3$ group $(p=0.056)$ (Figure 2A, 2D).

\section{High frequency of Tregs among CD4+ T cells} correlates with increased overall survival and is an independent prognostic factor in overall survival

The Treg ${ }^{\text {high }} /$ Total group showed significantly longer OS $(p=0.048)$ and a tendency of longer DFS compared to the Treglow/Total (Figure 1B, 1E). The Treghigh/ CD4 group showed longer OS $(p<0.001))$ and DFS $(p=0.039)$ than the Treg ${ }^{\text {low }} / \mathrm{CD} 4$ group (Figure 2B, $2 \mathrm{E}$ ) and remained a significant predictor of OS in the multivariate analysis (HR: 0.065 ; 95\% CI, 0.009-0.491; $p=0.008)$ (Table 4).
Low frequency of MDSCs among total examined cells correlates with increased overall survival and is an independent prognostic factor in overall survival

The MDSC ${ }^{\text {low }} /$ Total group was more frequent in old age patients and the proportion of MDSC ${ }^{\text {high }} /$ Total group decreased as the TNM stage advanced (Table 2). The $\mathrm{MDSC}^{\text {low}} /$ Total group showed a significantly longer OS $(p=0.027)$ and a tendency of longer DFS compared to the MDSC high/Total groups $(p=0.151)$ (Figure 1C, 1F). MDSC/Total was an independent prognostic factor (HR 8.601; 95\% CI, 1.240-59.678; $p=0.029$ ) in OS (Table 4). $\mathrm{MDSC}^{\text {high}} / \mathrm{CD} 45+$ group showed better DFS rate $(p=0.038)$ and a tendency of better OS than MDSC ${ }^{\text {low } /}$ CD45+ group $(p=0.142)$ (Figure $2 \mathrm{C}, 2 \mathrm{~F})$.

\section{DISCUSSION}

In this study, we explored the frequencies of various immune cell subsets in GC tissues by multicolor flow cytometry and evaluated the prognostic value of intratumoral CD8+ cytotoxic T cell, Treg and MDSC frequencies. We found that GC tissues contained significant proportions of immune cells including Tregs and MDSCs and the percentages of Tregs among $\mathrm{CD} 4+\mathrm{T}$ cells and of MDSCs among myeloid cells 
Table 2: Clinicopathologic features according to the proportions of $\mathrm{CD8}+\mathrm{T}$ cells, regulatory T cells, and myeloid derived suppressor cells among total cells

\begin{tabular}{|c|c|c|c|c|c|c|c|c|c|c|c|}
\hline & & \multirow{2}{*}{$\begin{array}{c}\text { Total } \\
n(\%)\end{array}$} & \multicolumn{2}{|c|}{ CD8/Total } & \multirow[b]{2}{*}{$p$} & \multicolumn{2}{|c|}{ Treg/Total } & \multirow[b]{2}{*}{$p$} & \multicolumn{2}{|c|}{ MDSC/Total } & \multirow[b]{2}{*}{$p$} \\
\hline & & & $\begin{array}{c}\text { Low } \\
n(\%)\end{array}$ & $\begin{array}{c}\text { High } \\
n(\%)\end{array}$ & & $\begin{array}{c}\text { Low } \\
n(\%)\end{array}$ & $\begin{array}{c}\text { High } \\
n(\%)\end{array}$ & & $\begin{array}{c}\text { Low } \\
n(\%)\end{array}$ & $\begin{array}{c}\text { High } \\
n(\%)\end{array}$ & \\
\hline \multirow[b]{2}{*}{ Age } & $\geq 50$ & $8(28.6)$ & $3(17.6)$ & $5(45.5)$ & 0.200 & $1(11.1)$ & $7(36.8)$ & 0.214 & $7(46.7)$ & $1(7.7)$ & 0.038 \\
\hline & $<50$ & $20(71.4)$ & $\begin{array}{c}14 \\
(82.4)\end{array}$ & $6(54.5)$ & & $8(88.9)$ & $12(63.2)$ & & $8(53.3)$ & $\begin{array}{c}12 \\
(92.3)\end{array}$ & \\
\hline \multirow[b]{2}{*}{ Gender } & Female & $10(35.7)$ & $4(23.5)$ & $6(54.5)$ & 0.125 & $2(22.2)$ & $8(42.1)$ & 0.417 & $7(46.7)$ & $3(23.1)$ & 0.254 \\
\hline & Male & $18(64.3)$ & $\begin{array}{c}13 \\
(76.5)\end{array}$ & $5(45.5)$ & & $7(77.8)$ & $11(57.9)$ & & $8(53.3)$ & $\begin{array}{c}10 \\
(76.9)\end{array}$ & \\
\hline \multirow{2}{*}{ Size } & $\geq 8 \mathrm{~cm}$ & $13(46.4)$ & $8(47.1)$ & $5(45.5)$ & $0.934^{1}$ & $5(55.6)$ & $8(42.10$ & 0.689 & $7(46.7)$ & $6(46.2)$ & 1 \\
\hline & $<8 \mathrm{~cm}$ & $15(53.6)$ & $9(52.9)$ & $6(54.5)$ & & $4(44.4)$ & $11(57.9)$ & & $8(53.3)$ & $7(53.8)$ & \\
\hline \multirow{2}{*}{$\begin{array}{l}\text { Differen- } \\
\text { tiation }\end{array}$} & $\begin{array}{l}\text { W/D, } \\
M / D\end{array}$ & $10(35.7)$ & $8(47.1)$ & $2(18.2)$ & 0.226 & $5(55.6)$ & $5(26.3)$ & 0.210 & $6(40.0)$ & $4(30.8)$ & 0.705 \\
\hline & $\mathrm{P} / \mathrm{D}$ & $18(64.3)$ & $9(52.9)$ & $9(81.8)$ & & $4(44.4)$ & $14(73.7)$ & & $9(60.0)$ & $9(69.2)$ & \\
\hline \multirow[t]{2}{*}{ Lauren } & Intestinal & $15(53.6)$ & $\begin{array}{c}10 \\
(58.8) \\
\end{array}$ & $5(45.5)$ & $0.488^{1}$ & $7(77.8)$ & $8(42.1)$ & 0.114 & $9(60.0)$ & $6(46.2)$ & 0.705 \\
\hline & Diffuse & $13(46.4)$ & $7(41.2)$ & $6(54.5)$ & & $2(22.2)$ & $11(57.9)$ & & $6(40.0)$ & $7(53.8)$ & \\
\hline \multirow{3}{*}{ T stage } & 2 & $1(3.6)$ & $0(0)$ & $1(9.1)$ & 0.642 & $0(0)$ & $1(5.3)$ & 0.194 & $1(6.7)$ & $0(0)$ & 1 \\
\hline & 3 & $9(32.1)$ & $6(35.3)$ & $3(27.3)$ & & $5(55.6)$ & $4(21.1)$ & & $5(33.3)$ & $4(30.8)$ & \\
\hline & 4 & $18(64.3)$ & $\begin{array}{c}11 \\
(64.7) \\
\end{array}$ & 7 (63.6) & & $4(44.4)$ & $14(73.7)$ & & $9(60.0)$ & $9(69.2)$ & \\
\hline \multirow{4}{*}{ N stage } & 0 & $2(7.1)$ & $0(0)$ & $2(18.2)$ & $0.263^{2}$ & $0(0)$ & $2(10.5)$ & $0.456^{2}$ & $1(6.7)$ & $1(7.7)$ & $0.195^{2}$ \\
\hline & 1 & $4(14.3)$ & $2(11.8)$ & $1(9.1)$ & & $0(0)$ & $3(15.8)$ & & $0(0)$ & $3(23.1)$ & \\
\hline & 2 & $7(25.0)$ & $5(29.4)$ & $3(27.3)$ & & $4(44.4)$ & $4(21.1)$ & & $4(26.7)$ & $4(30.8)$ & \\
\hline & 3 & $15(53.6)$ & $\begin{array}{c}10 \\
(58.8)\end{array}$ & $5(45.5)$ & & $5(55.6)$ & $10(52.6)$ & & $\begin{array}{c}10 \\
(66.7)\end{array}$ & $5(38.5)$ & \\
\hline \multirow{5}{*}{$\begin{array}{l}\text { TNM } \\
\text { stage }\end{array}$} & IIA, IIB & $3(10.7)$ & $0(0)$ & $3(27.3)$ & $0.781^{2}$ & $0(0)$ & $3(15.8)$ & $0.846^{2}$ & $1(6.7)$ & $2(15.4)$ & $0.033^{2}$ \\
\hline & IIIA & $4(14.3)$ & $4(23.5)$ & $0(0)$ & & $1(11.1)$ & $3(15.8)$ & & $1(6.7)$ & $3(23.1)$ & \\
\hline & IIIB & $7(25.0)$ & $6(35.3)$ & $1(9.1)$ & & $6(66.7)$ & $1(5.3)$ & & $3(20.0)$ & $4(30.8)$ & \\
\hline & IIIC & $10(35.7)$ & $6(35.3)$ & $4(36.4)$ & & $1(11.1)$ & $9(47.4)$ & & $6(40.0)$ & $4(30.8)$ & \\
\hline & IV & $4(14.3)$ & $1(5.9)$ & $3(27.3)$ & & $1(11.1)$ & $3(15.8)$ & & $4(26.7)$ & $0(0)$ & \\
\hline \multirow{2}{*}{$\begin{array}{l}\text { Recur- } \\
\text { rence }\end{array}$} & No & 15 (53.6) & $7(41.2)$ & $8(72.7)$ & $0.102^{1}$ & $3(33.3)$ & $12(63.2)$ & 0.228 & $\begin{array}{c}10 \\
(66.7)\end{array}$ & $5(38.5)$ & $0.136^{1}$ \\
\hline & Yes & $13(46.4)$ & $\begin{array}{c}10 \\
(58.8)\end{array}$ & $3(27.3)$ & & $6(66.7)$ & $7(36.8)$ & & $5(33.3)$ & $8(61.5)$ & \\
\hline \multirow[t]{2}{*}{ Death } & No & $19(67.9)$ & $\begin{array}{c}10 \\
(58.8) \\
\end{array}$ & $9(81.8)$ & 0.249 & $4(44.4)$ & $15(78.9)$ & 0.097 & $\begin{array}{c}13 \\
(86.7) \\
\end{array}$ & $6(46.2)$ & 0.042 \\
\hline & Yes & $9(32.1)$ & $7(41.2)$ & $2(18.2)$ & & $5(55.6)$ & $4(21.1)$ & & $2(13.3)$ & 7 (53.8) & \\
\hline
\end{tabular}

${ }^{1}$ By Chi-Square test, ${ }^{2}$ By Cochran Armitage test, otherwise by Fisher's exact test.

Bold letter represent statistics having significance with $p<0.05$. 
Table 3: Clinicopathologic features according to the proportions of CD8+ $\mathbf{T}$ cells among CD3+ $T$ cells, regulatory $T$ cells among $C D 4+$ helper $T$ cells, and myeloid derived suppressor cells among CD45+ leukocytes

\begin{tabular}{|c|c|c|c|c|c|c|c|c|c|c|c|}
\hline & & \multirow[b]{2}{*}{ Total } & \multicolumn{2}{|c|}{ CD8/CD3 } & \multirow[b]{2}{*}{$p$} & \multicolumn{2}{|c|}{ Treg/CD4 } & \multirow[b]{2}{*}{$p$} & \multicolumn{2}{|c|}{ MDSC/CD45 } & \multirow[b]{2}{*}{$p$} \\
\hline & & & $\begin{array}{c}\text { Low } \\
n(\%)\end{array}$ & $\begin{array}{l}\text { High } \\
n(\%)\end{array}$ & & $\begin{array}{c}\text { Low } \\
n(\%)\end{array}$ & $\begin{array}{l}\text { High } \\
n(\%)\end{array}$ & & $\begin{array}{c}\text { Low } \\
n(\%)\end{array}$ & $\begin{array}{l}\text { High } \\
n(\%)\end{array}$ & \\
\hline \multirow[b]{2}{*}{ Age } & $\geq 50$ & $8(28.6)$ & $0(0)$ & $8(33.3)$ & 0.295 & $2(25.0)$ & $6(30.0)$ & 1.000 & $3(37.5)$ & $5(25.0)$ & 0.651 \\
\hline & $<50$ & $20(71.4)$ & $4(100)$ & $16(66.7)$ & & $6(75.0)$ & $14(70.0)$ & & $5(62.5)$ & $\begin{array}{c}15 \\
(75.0) \\
\end{array}$ & \\
\hline \multirow[b]{2}{*}{ Gender } & Female & $10(35.7)$ & $0(0)$ & $10(41.7)$ & 0.265 & $2(25.0)$ & $8(40.0)$ & 0.669 & $4(50.0)$ & $6(30.0)$ & 0.400 \\
\hline & Male & $18(64.3)$ & $4(100)$ & $14(58.3)$ & & $6(75.0)$ & $12(60.0)$ & & $4(50.0)$ & $\begin{array}{c}14 \\
(70.0) \\
\end{array}$ & \\
\hline \multirow[t]{2}{*}{ Size } & $\geq 8 \mathrm{~cm}$ & $13(46.4)$ & $0(0)$ & $13(54.2)$ & 0.102 & $4(50.0)$ & $9(45.0)$ & 1.000 & $2(25.0)$ & $\begin{array}{c}11 \\
(55.0) \\
\end{array}$ & 0.221 \\
\hline & $<8 \mathrm{~cm}$ & $15(53.6)$ & $4(100)$ & $11(45.8)$ & & $4(50.0)$ & $11(55.0)$ & & $6(75.0)$ & $9(45.0)$ & \\
\hline \multirow{2}{*}{$\begin{array}{l}\text { Differen- } \\
\text { tiation }\end{array}$} & $\begin{array}{l}\text { W/D, } \\
M / D\end{array}$ & $10(35.7)$ & $2(50.0)$ & $8(33.3)$ & 0.601 & $2(25.0)$ & $8(40.0)$ & 0.669 & $3(37.5)$ & $7(35.0)$ & 1.000 \\
\hline & $\mathrm{P} / \mathrm{D}$ & $18(64.3)$ & $2(50.0)$ & $16(66.7)$ & & $6(75.0)$ & $12(60.0)$ & & $5(62.5)$ & $\begin{array}{c}13 \\
(65.0) \\
\end{array}$ & \\
\hline \multirow[t]{2}{*}{ Lauren } & Intestinal & $15(53.6)$ & $3(75.0)$ & $12(50.0)$ & 0.600 & $5(62.5)$ & $10(50.0)$ & 0.686 & $4(50.0)$ & $\begin{array}{c}11 \\
(55.0)\end{array}$ & 1.000 \\
\hline & Diffuse & $13(46.4)$ & $1(25.0)$ & $12(50.0)$ & & $3(37.5)$ & $10(50.0)$ & & $4(50.0)$ & $9(45.0)$ & \\
\hline \multirow{3}{*}{ T stage } & 2 & $1(3.6)$ & $0(0)$ & $1(4.2)$ & $0.166^{1}$ & $0(0)$ & $1(5.0)$ & $0.915^{1}$ & $0(0)$ & $1(5.0)$ & $715^{1}$ \\
\hline & 3 & 1,9 & $3(75.0)$ & $6(25.0)$ & & $3(37.5)$ & $6(30.0)$ & & $4(50.0)$ & $5(25.0)$ & \\
\hline & 4 & $18(64.3)$ & $1(25.0)$ & $17(70.8)$ & & $5(62.5)$ & $13(65.0)$ & & $4(50.0)$ & $\begin{array}{c}14 \\
(70.0)\end{array}$ & \\
\hline \multirow{4}{*}{ N stage } & 0 & $2(7.1)$ & $1(25.0)$ & $1(4.2)$ & $0.023^{1}$ & $1(12.5)$ & $1(5.0)$ & 0.1861 & $1(12.5)$ & $1(5.0)$ & $0.833^{1}$ \\
\hline & 1 & $4(14.3)$ & $1(25.0)$ & $2(8.3)$ & & $1(12.5)$ & $2(10.0)$ & & $0(0)$ & $3(15.0)$ & \\
\hline & 2 & $7(25.0)$ & $1(25.0)$ & $7(29.2)$ & & $3(37.5)$ & $5(25.0)$ & & $2(25.0)$ & $6(30.0)$ & \\
\hline & 3 & $15(53.6)$ & $1(25.0)$ & $14(58.3)$ & & $3(37.5)$ & $12(60.0)$ & & $5(62.5)$ & $\begin{array}{c}10 \\
(50.0)\end{array}$ & \\
\hline \multirow{5}{*}{$\begin{array}{l}\text { TNM } \\
\text { stage }\end{array}$} & IIA, IIB & $3(10.7)$ & $0(0)$ & $3(12.5)$ & $0.604^{1}$ & $0(0)$ & $3(15.0)$ & $0.547^{1}$ & $0(0)$ & $3(15.0)$ & $0.123^{1}$ \\
\hline & IIIA & $4(14.3)$ & $2(50.0)$ & $2(8.3)$ & & $2(25.0)$ & $2(10.0)$ & & $1(12.5)$ & $3(15.0)$ & \\
\hline & IIIB & $7(25.0)$ & $1(25.0)$ & $6(25.0)$ & & $2(25.0)$ & $5(25.0)$ & & $2(25.0)$ & $5(25.0)$ & \\
\hline & IIIC & $10(35.7)$ & $0(0)$ & $10(41.7)$ & & $2(25.0)$ & $8(40.0)$ & & $2(25.0)$ & $8(40.0)$ & \\
\hline & IV & $4(14.3)$ & $1(25.0)$ & $3(12.5)$ & & $2(25.0)$ & $2(10.0)$ & & $3(37.5)$ & $1(5.0)$ & \\
\hline \multirow{2}{*}{$\begin{array}{l}\text { Recur- } \\
\text { rence }\end{array}$} & No & $15(53.6)$ & $1(25.0)$ & $14(58.3)$ & 0.311 & $2(25.0)$ & $13(65.0)$ & 0.096 & $2(25.0)$ & $\begin{array}{c}13 \\
(65.0) \\
\end{array}$ & 0.096 \\
\hline & Yes & $13(46.4)$ & $3(75.0)$ & $10(41.7)$ & & $6(75.0)$ & $7(35.0)$ & & $6(75.0)$ & $7(35.0)$ & \\
\hline \multirow[t]{2}{*}{ Death } & No & $19(67.9)$ & $1(25.0)$ & $18(75.0)$ & 0.084 & $2(25.0)$ & $17(85.0)$ & 0.005 & $4(50.0)$ & $\begin{array}{c}15 \\
(75.0) \\
\end{array}$ & 0.371 \\
\hline & Yes & $9(32.1)$ & $3(75.0)$ & $6(25.0)$ & & $6(75.0)$ & $3(15.0)$ & & $4(50.0)$ & $5(25.0)$ & \\
\hline
\end{tabular}

${ }^{1}$ By Cochran Armitage test, otherwise by Fisher's exact test.

Bold letter represent statistics having significance with $p<0.05$. 
were substantially higher when compared with those in peripheral blood from healthy individuals. Moreover, the high proportion of Tregs among CD4+ T cells was an independent prognostic factor for longer OS, whereas the increased proportion of MDSCs among total examined cells was associated with shorter OS.

Infiltration of CD8+ cytotoxic T cells into tumors has been reported as a favorable prognostic factor in many cancers including GCs [10-17]. Here, we report that higher frequencies of $\mathrm{CD} 8+\mathrm{T}$ cell among tumorinfiltrating CD3 $+\mathrm{T}$ cells are associated with longer OS. The prognostic significance of Tregs infiltration remains controversial and seems different depending on the specific tumor type. Many studies have shown that a high density of Tregs in IHC results is associated with a poor outcome, and these results were explained on the basis of the suppressive effects of Tregs on antitumor cytotoxic $T$ cells [24-26]. However, a few recent studies have highlighted the protective effects of Tregs, especially in gastric or colorectal cancers occurring in an inflammatory background caused by microorganisms $[9,14,28-30]$. In this context, Tregs were shown to inhibit the immune reaction of cytotoxic $\mathrm{T}$ cells in response to bacteria rather than cancer cells, and, thereby, to suppress cancer progression by controlling inflammation. In GC, the results of several studies on TILs focused on Tregs are controversial [9, 28, 31-33]. This discrepancy could be explained by differences in technical issue; specific FOXP3 antibodies, scoring or statistical process. Moreover, most of the previous studies defined Tregs as FoxP3+ cells by IHC. Unlike previous studies using a single marker to detect a specific type of immune cell by IHC followed by manual quantification of positive cells, we used flow cytometry to analyze the cell populations at the single-cell level. This approach made possible to identify cell lineages or subsets by using multiple monoclonal antibodies at the same time and utilizing objective quantification with an automated system. So, overview of the immune cell population from the cancer tissue was possible and we determined the percentage of immune cells among the total number of examined cells as well as the proportion of each immune cells among their belonging subsets of immune cells.

In this study, we could define tumor infiltrating Tregs in detail as CD45+CD4+CD25 \pm and FOXP3+ cells
A

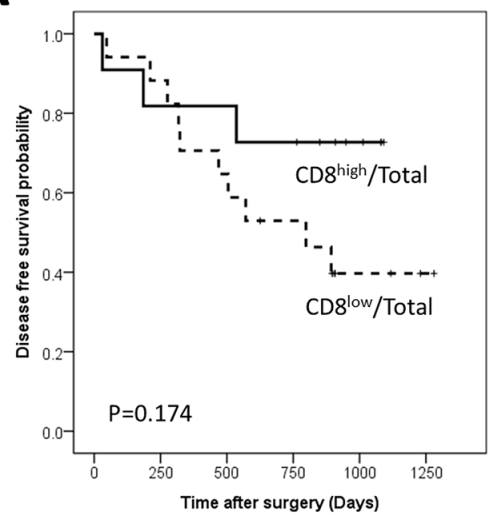

D

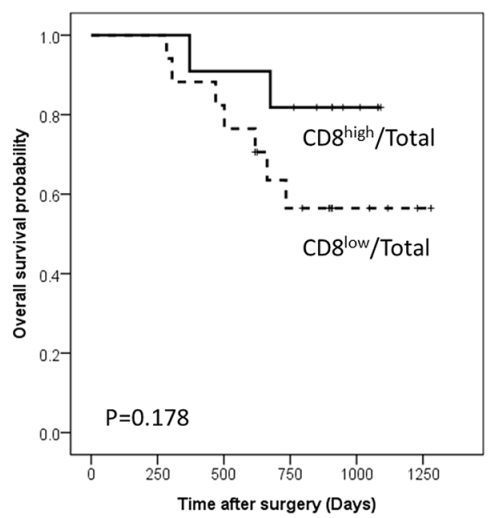

B

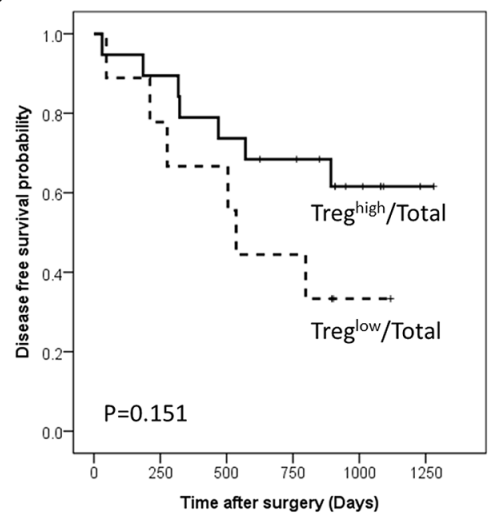

$\mathbf{E}$

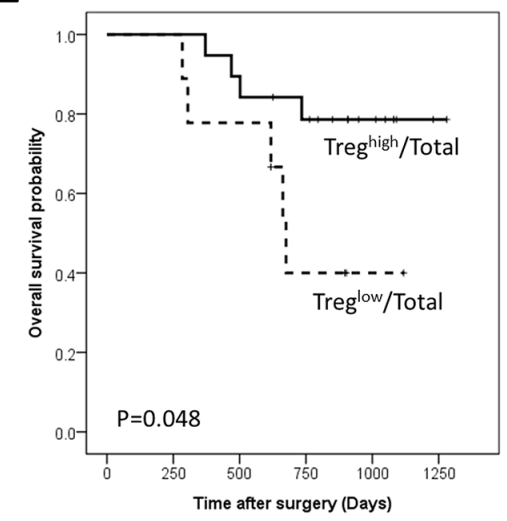

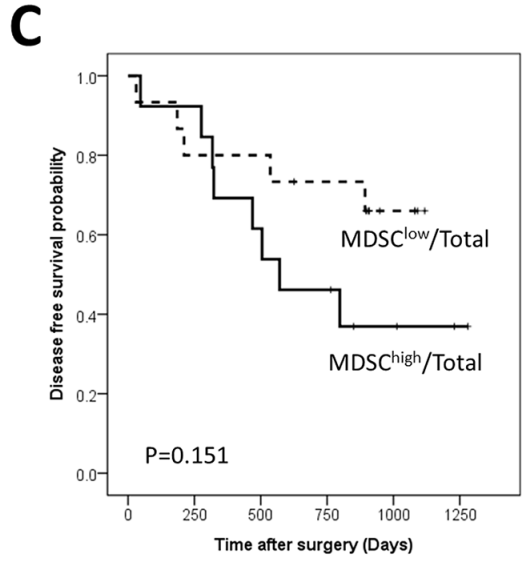

$\mathbf{F}$

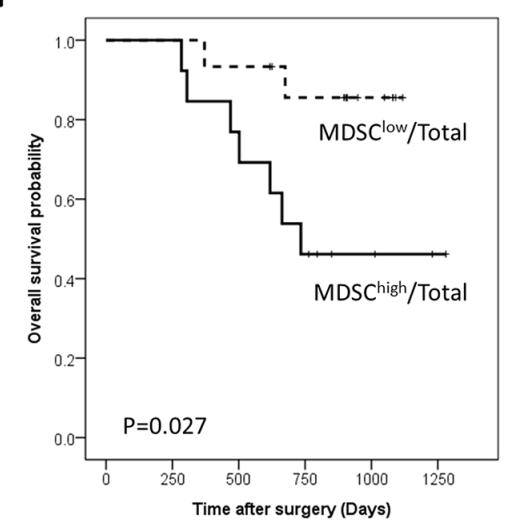

Figure 1: Disease-free and overall survival curves of gastric cancer patients according to the proportions of CD8+ $T$ cells $(A, D)$, regulatory $T$ cells (Tregs) $(B, E)$ and Myeloid derived suppressor cells (MDSCs) $(C, F)$ among total examined cells. 
and found that a higher frequency of Tregs among total examined cells was associated with a longer OS, and this result is in line with several prior reports $[9,33]$. Furthermore, we found that the proportion of Tregs among CD4+ T cells was an independent favorable prognostic factor, which could not be evaluated by traditional IHC method. In addition to traditional concept of Tregs suppressing antitumor cytotoxic T cells, several other subsets of Treg have been identified, and direct tumor-killing Tregs activity has been reported [34, 35]. The functional relevance of Treg-mediated tumor killing remains unknown. Further functional studies of Tregs may elucidate their role in the antitumor response and may help to explain the observed associations with the disease prognosis.

MDSCs are generally defined based on the combined expression of several markers and cannot simply be identified by IHC in cancer tissue. Therefore, MDSCs have been rarely studied in cancer tissue. Recently, Sun et al. reported that CD33-HLA-DR-MDSC were increased in the peripheral blood and tumor tissue of colorectal cancer patients compared to the peripheral blood of healthy controls [36]. They also found that an increased frequency of MDSCs was correlated with nodal/distant metastasis and tumor stage. In GC, two previous studies showed increased MDSCs in PBMCs from cancer patients and that MDSC frequency was associated with adverse clinical outcomes $[37,38]$. In the present study, we found that high MDSC percentages among total cells were an independent adverse prognostic factor in patients with advanced GC. This result may be explained by the role of MDSCs as inhibitors of antitumor immunity [39-42].

Although this prospective study provides valuable insights on de novo presence and relative quantity of multiple immune cell subsets in GC tissues, current study has limitations as follow: First, it was performed in relatively small number of patients with short follow up period. Second, the cutoffs, which were determined with the highest statistical significance for patient survival in presents study, need to be validated in an independent cohort. Third, the location of the immune cells within the tumor remains unknown whether the immune cells are in the intraepithelial, intratumoral or peritumoral stroma. To improve our understanding of the complex role of immune cells in GC, further studies combining both flow cytometry and IHC on a larger number of cases are necessary.

In conclusion, we have demonstrated that high levels of Tregs among tumor-infiltrating CD4+ T cells
A

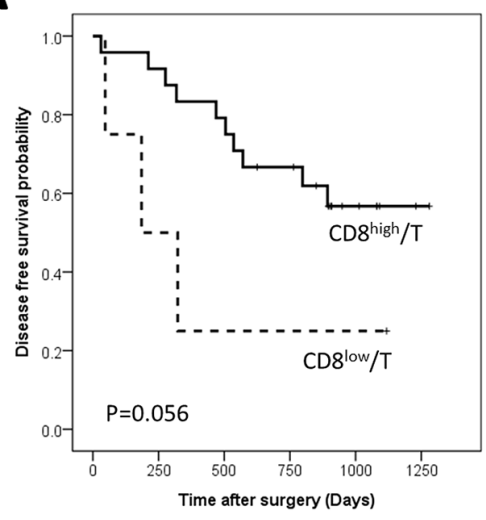

D



B

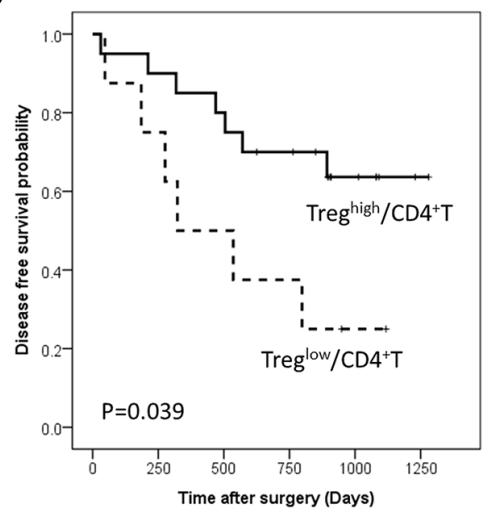

E

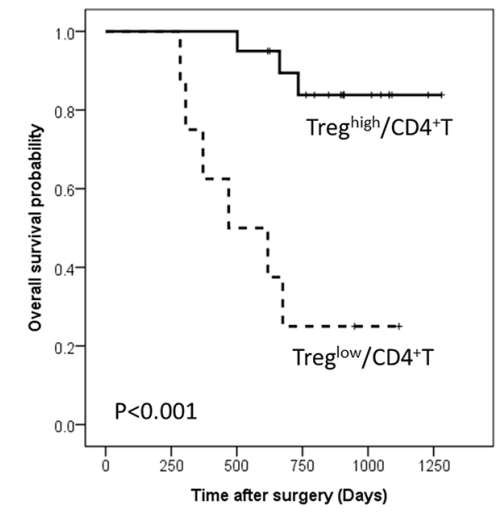

C

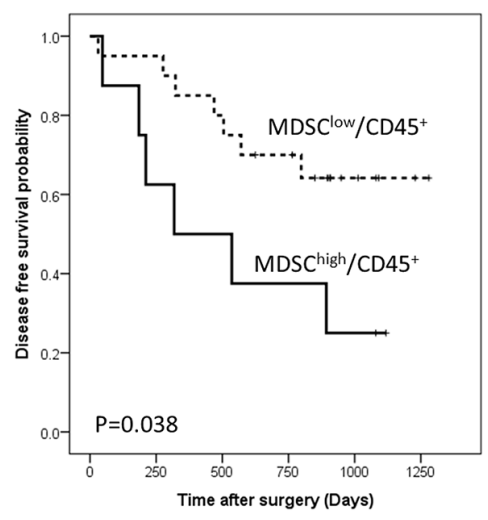

$\mathbf{F}$

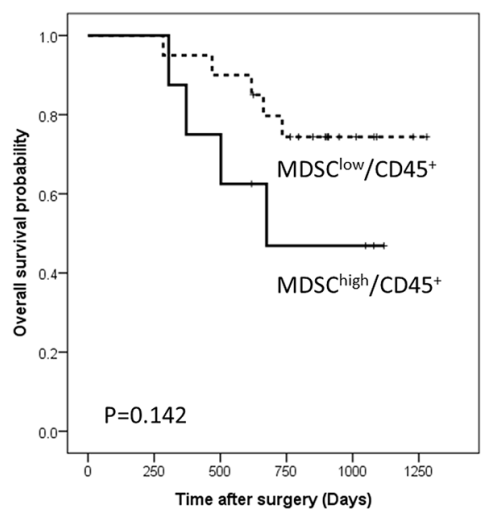

Figure 2: Disease-free and overall survival curves of gastric cancer patients according to the proportions of CD8+ $\mathrm{T}$ cells among CD3 + $T$ cells $(A, D)$, regulatory $T$ cells (Tregs) among CD4+ $T$ cells $(B, E)$, and Myeloid derived suppressor cells (MDSCs) among CD45+ leukocytes (C, F). 
Table 4: The results of univariate and multivariate analyses

\begin{tabular}{|c|c|c|c|c|c|c|c|}
\hline & & \multicolumn{6}{|c|}{ Disease free survival } \\
\hline & & \multicolumn{3}{|c|}{ Univariate } & \multicolumn{3}{|c|}{ Multivariate } \\
\hline & & HR & $95 \% \mathrm{CI}$ & $p$ value & HR & $95 \% \mathrm{CI}$ & $p$ value \\
\hline Age & $\geq 50 \mathrm{vs}<50$ & 3.153 & $0.696-14.295$ & 0.136 & 1.767 & $0.224-13.974$ & 0.589 \\
\hline Gender & Male vs female & 1.504 & $0.462-4.895$ & 0.498 & & & \\
\hline Size & $\geq 8 \mathrm{~cm} \mathrm{vs}<8 \mathrm{~cm}$ & 1.870 & $0.608-5.751$ & 0.275 & 2.059 & $0.605-7.013$ & 0.248 \\
\hline Lauren & $\begin{array}{l}\text { Diffuse or mixed vs } \\
\text { intestinal }\end{array}$ & 1.302 & $0.437-3.879$ & 0.635 & & & \\
\hline Differentiation & $\mathrm{P} / \mathrm{D}$ vs $\mathrm{W} / \mathrm{D}$ or $\mathrm{M} / \mathrm{D}$ & 0.767 & $0.250-2.349$ & 0.642 & & & \\
\hline TNM stage & IIIB-IV vs II, IIIA & 2.318 & $0.513-10.472$ & 0.275 & 2.63 & $0.529-13.083$ & 0.238 \\
\hline CD8/Total & High vs low & 0.419 & $0.115-1.527$ & 0.188 & 0.933 & $0.173-5.049$ & 0.936 \\
\hline Treg/Total & High vs low & 0.458 & $0.153-1.367$ & 0.162 & 0.481 & $0.124-1.866$ & 0.290 \\
\hline MDSC/Total & High vs low & 2.246 & $0.724-6.695$ & 0.161 & 2.171 & $0.573-8.222$ & 0.254 \\
\hline Age & $\geq 50$ vs $<50$ & 3.153 & $0.696-14.295$ & 0.136 & 8.987 & $1.275-63.359$ & 0.028 \\
\hline Gender & Male vs female & 1.504 & $0.462-4.895$ & 0.498 & & & \\
\hline Size & $\geq 8 \mathrm{~cm} \mathrm{vs}<8 \mathrm{~cm}$ & 1.870 & $0.608-5.751$ & 0.275 & 1.659 & $0.299-9.208$ & 0.563 \\
\hline Lauren & $\begin{array}{l}\text { Diffuse or mixed vs } \\
\text { intestinal }\end{array}$ & 1.302 & $0.437-3.879$ & 0.635 & & & \\
\hline Differentiation & $\mathrm{P} / \mathrm{D}$ vs $\mathrm{W} / \mathrm{D}$ or $\mathrm{M} / \mathrm{D}$ & 0.767 & $0.250-2.349$ & 0.642 & & & \\
\hline TNM stage & IIIB-IV vs II, IIIA & 2.318 & $0.513-10.472$ & 0.275 & 7.271 & $0.788-67.136$ & 0.080 \\
\hline $\mathrm{CD} 8 / \mathrm{CD} 3$ & High vs low & 0.300 & $0.081-1.110$ & 0.071 & 1.535 & $0.074-31.937$ & 0.782 \\
\hline Treg/CD4 & High vs low & 0.331 & $0.110-0.994$ & 0.049 & 0.259 & $0.042-1.618$ & 0.149 \\
\hline \multirow[t]{4}{*}{ MDSC/CD45 } & High vs low & 3.024 & $1.010-9.055$ & 0.048 & 5.151 & $0.938-28.292$ & 0.059 \\
\hline & & \multicolumn{6}{|c|}{ Overall survival } \\
\hline & & \multicolumn{3}{|c|}{ Univariate } & \multicolumn{3}{|c|}{ Multivariate } \\
\hline & & HR & $95 \% \mathrm{CI}$ & $p$ value & HR & $95 \%$ CI & $p$ value \\
\hline Age & $\geq 50$ vs $<50$ & 3.898 & $0.487-31.205$ & 0.200 & 0.976 & $0.076-12.597$ & 0.985 \\
\hline Gender & Male vs female & 1.175 & $0.293-4.707$ & 0.820 & & & \\
\hline Size & $\geq 8 \mathrm{~cm} \mathrm{vs}<8 \mathrm{~cm}$ & 1.915 & $0.478-7.670$ & 0.359 & & & \\
\hline Lauren & $\begin{array}{l}\text { Diffuse or mixed vs } \\
\text { intestinal }\end{array}$ & 1.267 & $0.340-4.722$ & 0.724 & & & \\
\hline Differentiation & $\mathrm{P} / \mathrm{D}$ vs $\mathrm{W} / \mathrm{D}$ or $\mathrm{M} / \mathrm{D}$ & 0.958 & $0.239-3.846$ & 0.952 & & & \\
\hline TNM stage & IIIB-IV vs II, IIIA & 3.119 & $0.389-24.990$ & 0.284 & 3.603 & $0.392-33.125$ & 0.257 \\
\hline CD8/Total & High vs low & 0.354 & $0.073-1.713$ & 0.197 & 1.087 & $0.164-7.199$ & 0.931 \\
\hline Treg/Total & High vs low & 0.284 & $0.075-1.070$ & 0.063 & 0.226 & $0.043-1.181$ & 0.078 \\
\hline MDSC/Total & High vs low & 4.955 & $1.027-23.897$ & 0.046 & 8.601 & $1.240-59.678$ & 0.029 \\
\hline Age & $\geq 50$ vs $<50$ & 3.898 & $0.487-31.205$ & 0.200 & 21.658 & $0.941-498.647$ & 0.055 \\
\hline Gender & Male vs female & 1.175 & $0.293-4.707$ & 0.820 & & & \\
\hline Size & $\geq 8 \mathrm{~cm} \mathrm{vs}<8 \mathrm{~cm}$ & 1.915 & $0.478-7.670$ & 0.359 & & & \\
\hline Lauren & $\begin{array}{l}\text { Diffuse or mixed vs } \\
\text { intestinal }\end{array}$ & 1.267 & $0.340-4.722$ & 0.724 & & & \\
\hline Differentiation & $\mathrm{P} / \mathrm{D}$ vs $\mathrm{W} / \mathrm{D}$ or $\mathrm{M} / \mathrm{D}$ & 0.958 & $0.239-3.846$ & 0.952 & & & \\
\hline TNM stage & IIIB-IV vs II, IIIA & 3.119 & $0.389-24.990$ & 0.284 & 24.216 & $1.131-518.706$ & 0.042 \\
\hline $\mathrm{CD} 8 / \mathrm{CD} 3$ & High vs low & 0.164 & $0.039-0.684$ & 0.013 & 0.786 & $0.021-29.689$ & 0.897 \\
\hline
\end{tabular}




\begin{tabular}{|l|l|c|c|c|c|c|c|}
\hline Treg/CD4 & High vs low & $\mathbf{0 . 1 1 3}$ & $\mathbf{0 . 0 2 8}-\mathbf{0 . 4 6 0}$ & $\mathbf{0 . 0 0 2}$ & $\mathbf{0 . 0 4 7}$ & $\mathbf{0 . 0 0 6}-\mathbf{0 . 3 7 2}$ & $\mathbf{0 . 0 0 4}$ \\
\hline MDSC/CD45 & High vs low & 2.598 & $0.692-9.755$ & 0.157 & 2.477 & $0.186-32.892$ & 0.492 \\
\hline
\end{tabular}

were favorable, but an increased proportion of MDSCs was an adverse independent prognostic factor in GC. Our results may provide important insights for further classification of GC based on immunological feature and also for future immunotherapy in GC.

\section{MATERIALS AND METHODS}

\section{Patients}

Tissue samples from 28 patients with GC undergoing curative resection of the primary tumor at the Department of Surgery at the Samsung Medical Center between June 2010 and September 2011 were included in this study (18 males, 10 females; ages ranging from 37 to 88 years). The research protocol was approved by the Institutional Review Board. Written informed consent was received from all patients. Tumor stage and grading were classified according to the 7th edition of the TNM Classification of the International Union Against Cancer [43]. Patients with other malignant diseases in their medical history and patients treated with neoadjuvant chemotherapy were excluded from this study. Six patients had postoperative concurrent chemo-radiation therapy and 13 patients performed postoperative chemotherapy. The median follow-up period was 29 months (range 10-42 months).

Tumor tissues were taken from the center of the cancer immediately after surgical removal and histologic confirmation. For preparation of single cell suspensions, tumor samples were mechanically dissected and washed with PBS. The cell suspension was passed through a sterile $40 \mu \mathrm{m}$ nylon filter (BD Falcon, Heidelberg, Germany), and single cells were pelleted and suspended in PBS. Data from peripheral blood mononuclear cells from 8 healthy individuals were used as a control.

\section{Multicolor flow cytometry}

Multicolor immunofluorescence and multiparameter flow cytometric analyses were used to characterize the single-cell suspensions. To distinguish

\section{A. Regulatory $\mathrm{T}$ cells}

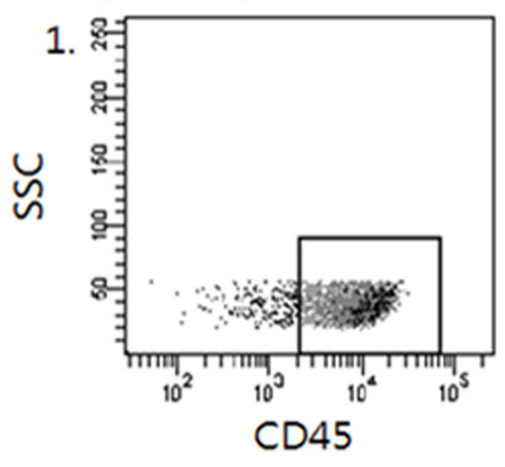

B. MDSC

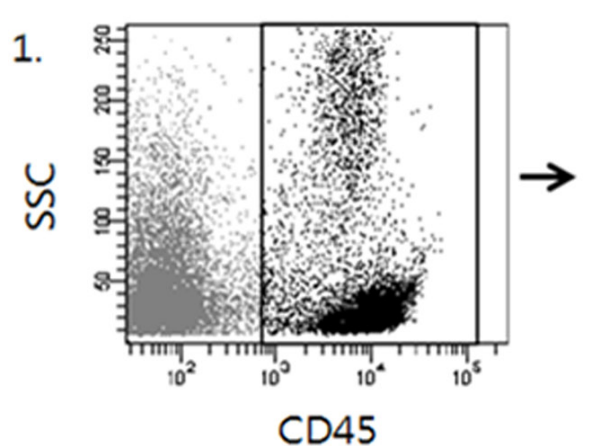

CD45
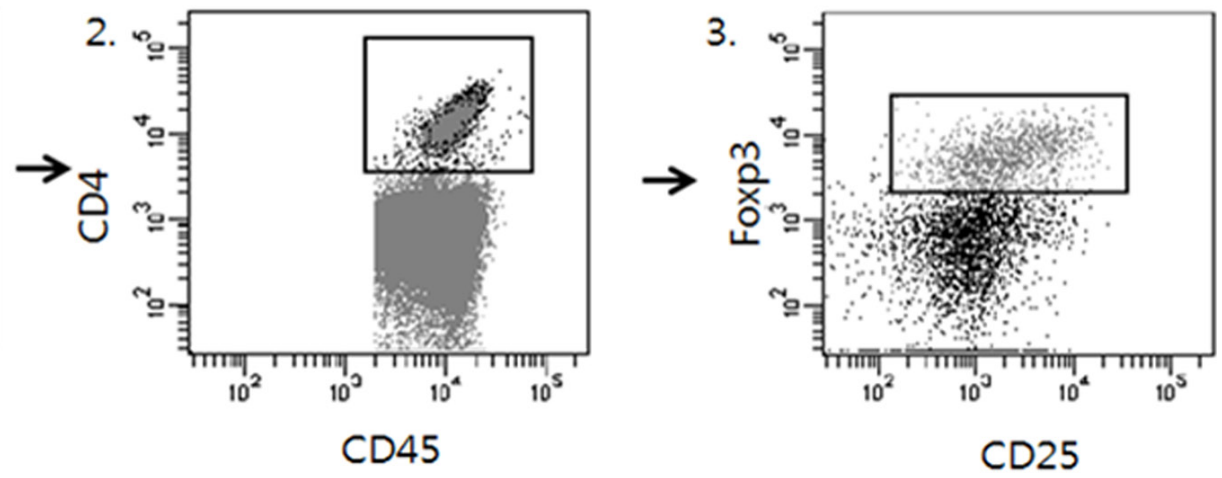

CD25

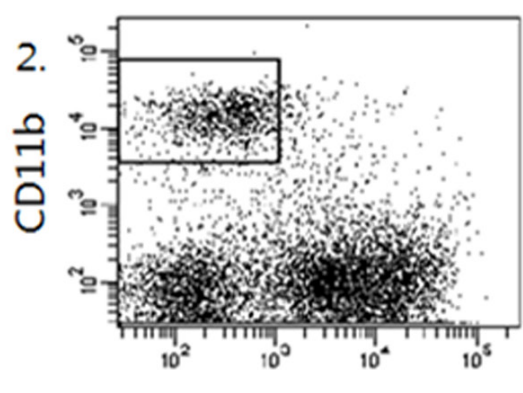

HLA-DR
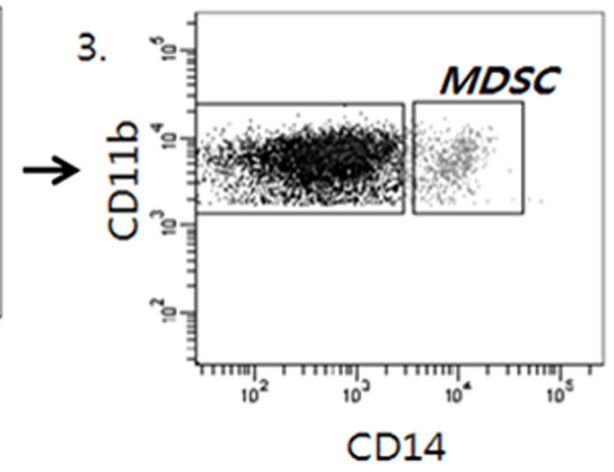

Figure 3: Analysis strategy to detect regulatory T cells (Tregs) and Myeloid derived suppressor cells (MDSCs) in tumor tissues by multicolor flow cytometry. Dot plots of representative patient are displayed. Multiple antibodies were used to sort out the appropriate immune cell lineages among tumor cells. A. Tregs were defined as CD45+CD4+CD25 \pm and FOXP3+. B. MDSCs were defined as CD45+, CD11b+, HLA-DR- and CD14+. 
TILs from the tumor cells, initial gating on anti-CD45 (2D1; BD Biosciences, San Jose, CA) positive cells was performed. Afterwards, anti-CD3 (SK7; BD Biosciences, San Jose, CA), anti-CD4 (RPA-T4; eBioscience, San Diego, CA), anti-CD8 (PRA-T8; BD Biosciences, San Jose, CA), anti-CD25 (BC96; eBioscience), and antiFoxp3 (PCH101; eBioscience) were applied for the identification of the $\mathrm{T}$ cell subsets. To detect the NK subset, anti-CD56 (MEM188; eBioscience) and anti-CD16 (CB16; eBioscience) were applied. Finally, to identify MDSCs, anti-HLA-DR (LN3; eBioscience), anti-CD11b (ICRF44; eBioscience), anti-CD14 (61D3; eBioscience) and 7-AAD (eBioscience) for live cell gating were used. The unstained cells and cells stained with relevant isotype antibodies were used as controls. The antibodies listed above are all mouse anti-human monoclonal antibodies.

The cells were incubated with the surface marker antibodies for $30 \mathrm{~min}$ at $4^{\circ} \mathrm{C}$ and washed twice with PBS. To conduct intracellular staining, $1 \mathrm{ml}$ of fixation and permeabilization mixture (eBioscience) was added, and the cells were incubated on ice for an hour. After incubation, the cells were washed twice with PBS. After washing, the cells were mixed with the antibodies and incubated at room temperature for 15 minutes. After the final wash, the cells were resuspended in PBS buffer and analyzed with a FACS Canto II Flow Cytometer (BD Biosciences; FACS DIVA software ver. 6.1.3).

\section{Data analysis}

We defined helper T cells as CD45+ CD3+ CD4+, cytotoxic T cells as CD45+ CD3+ CD8+, Tregs as CD45+ CD4+ CD25 \pm and FOXP3+, and MDSCs as CD45+ CD11b+ HLA-DR- and CD14+ [21, 22, 27, 44-48] (Figure 3). The frequency of each cell subgroup among the total examined cells was determined. We also assessed the percentages of the following immune cell subsets among leukocytes: CD8+ T cells among CD3+ T cells, Tregs among CD4+ T cells, and MDSCs among total CD45+ leukocytes. To identify optimal cutoffs with the highest statistical significance for patient survival, we used X-Tile statistical software (Yale University, New Haven, CT, USA) [49] and grouped the cases into low- and highdensity groups for each marker following dichotomization.

\section{Statistics}

Pearson's chi-square test, Fisher's exact test, and the Cochran-Armitage test were used as appropriate for statistical analysis. Disease-free survival (DFS) was defined as the time from surgery to the first relapse of cancer, occurrence of a second primary tumor, or death from any cause. Overall survival (OS) was measured from the date of surgery to the date of death. The OS and DFS were calculated using the Kaplan-Meier method, and survival curves were compared by the log-rank method.
The Cox proportional hazard model was used to evaluate the association between clinicopathologic factors and survival for multivariate analysis. In the multivariate analysis, variables showing $p$ values of less than 0.3 in the univariate analysis were included as covariates. All statistical analyses were performed using SPSS software (SPSS Inc., Chicago, IL, USA) or R software (version 3.03 ), and $p$ values less than 0.05 were considered to be statistically significant.

\section{ACKNOWLEGMENTS}

We thank Professor Kyung-A Kim at Biostatistics and Clinical Epidemiology Center, Research Institute for Future Medicine, Samsung Medical Center for her statistical advice.

\section{GRANT SUPPORT}

This work was supported by Basic Science Research Program through the National Research Foundation of Korea (NRF) funded by the Ministry of Education (NRF- 2012R1A1A3015504) and Samsung Biomedical Research Institute (SBRI-SMX1132721 and C-B0-301).

\section{CONFLICTS OF INTEREST}

The authors declare no conflicts of interest.

\section{REFERENCES}

1. Kamangar F, Dores GM, Anderson WF. Patterns of cancer incidence, mortality, and prevalence across five continents: defining priorities to reduce cancer disparities in different geographic regions of the world. J Clin Oncol. 2006; 24:2137-2150.

2. Kelley JR, Duggan JM. Gastric cancer epidemiology and risk factors. J Clin Epidemiol. 2003; 56:1-9.

3. Lee J, Lim do H, Kim S, Park SH, Park JO, Park YS, Lim HY, Choi MG, Sohn TS, Noh JH, Bae JM, Ahn YC, Sohn I, et al. Phase III trial comparing capecitabine plus cisplatin versus capecitabine plus cisplatin with concurrent capecitabine radiotherapy in completely resected gastric cancer with D2 lymph node dissection: the ARTIST trial. J Clin Oncol. 2012; 30:268-273.

4. DeNardo DG, Coussens LM. Inflammation and breast cancer. Balancing immune response: crosstalk between adaptive and innate immune cells during breast cancer progression. Breast Cancer Res. 2007; 9:212.

5. Galon J, Costes A, Sanchez-Cabo F, Kirilovsky A, Mlecnik B, Lagorce-Pages C, Tosolini M, Camus M, Berger A, Wind P, Zinzindohoue F, Bruneval P, Cugnenc PH, et al. Type, density, and location of immune cells within human colorectal tumors predict clinical outcome. Science. 2006; 313:1960-1964. 
6. Leffers N, Gooden MJ, de Jong RA, Hoogeboom BN, ten Hoor KA, Hollema H, Boezen HM, van der Zee AG, Daemen T, Nijman HW. Prognostic significance of tumorinfiltrating T-lymphocytes in primary and metastatic lesions of advanced stage ovarian cancer. Cancer Immunol Immunother. 2009; 58:449-459.

7. Sato E, Olson SH, Ahn J, Bundy B, Nishikawa H, Qian F, Jungbluth AA, Frosina D, Gnjatic S, Ambrosone C, Kepner J, Odunsi T, Ritter $\mathrm{G}$, et al. Intraepithelial CD8+ tumorinfiltrating lymphocytes and a high $\mathrm{CD} 8+$ /regulatory $\mathrm{T}$ cell ratio are associated with favorable prognosis in ovarian cancer. Proc Natl Acad Sci USA. 2005; 102:18538-18543.

8. Zhang L, Conejo-Garcia JR, Katsaros D, Gimotty PA, Massobrio M, Regnani G, Makrigiannakis A, Gray H, Schlienger K, Liebman MN, Rubin SC, Coukos G. Intratumoral $\mathrm{T}$ cells, recurrence, and survival in epithelial ovarian cancer. N Engl J Med. 2003; 348: 203-213.

9. Kim KJ, Lee KS, Cho HJ, Kim YH, Yang HK, Kim WH, Kang GH. Prognostic implications of tumor-infiltrating FoxP3+ regulatory $\mathrm{T}$ cells and $\mathrm{CD} 8+$ cytotoxic $\mathrm{T}$ cells in microsatellite-unstable gastric cancers. Hum Pathol. 2014; 45:285-293.

10. Kondratiev S, Sabo E, Yakirevich E, Lavie O, Resnick MB. Intratumoral CD8+ T lymphocytes as a prognostic factor of survival in endometrial carcinoma. Clin Cancer Res. 2004; 10:4450-4456.

11. Lee HE, Chae SW, Lee YJ, Kim MA, Lee HS, Lee BL, Kim WH. Prognostic implications of type and density of tumour-infiltrating lymphocytes in gastric cancer. $\mathrm{Br} \mathrm{J}$ Cancer. 2008; 99:1704-1711.

12. Oshikiri T, Miyamoto M, Shichinohe T, Suzuoki M, Hiraoka K, Nakakubo Y, Shinohara T, Itoh T, Kondo S, Katoh H. Prognostic value of intratumoral CD8+ T lymphocyte in extrahepatic bile duct carcinoma as essential immune response. J Surg Oncol. 2003; 84:224-228.

13. Schumacher K, Haensch W, Roefzaad C, Schlag PM. Prognostic significance of activated $\mathrm{CD} 8(+) \mathrm{T}$ cell infiltrations within esophageal carcinomas. Cancer Res. 2001; 61:3932-3936.

14. Sharma P, Shen Y, Wen S, Yamada S, Jungbluth AA, Gnjatic S, Bajorin DF, Reuter VE, Herr H, Old LJ and Sato E. CD8 tumor-infiltrating lymphocytes are predictive of survival in muscle-invasive urothelial carcinoma. Proc Natl Acad Sci USA. 2007; 104:3967-3972.

15. Al-Shibli K, Al-Saad S, Donnem T, Persson M, Bremnes RM, Busund LT. The prognostic value of intraepithelial and stromal innate immune system cells in non-small cell lung carcinoma. Histopathology. 2009; 55:301-312.

16. Dai F, Liu L, Che G, Yu N, Pu Q, Zhang S, Ma J, Ma L, You Z. The number and microlocalization of tumorassociated immune cells are associated with patient's survival time in non-small cell lung cancer. BMC Cancer. 2010; 10:220.
17. Mlecnik B, Tosolini M, Kirilovsky A, Berger A, Bindea G, Meatchi T, Bruneval P, Trajanoski Z, Fridman WH, Pages F, Galon J. Histopathologic-based prognostic factors of colorectal cancers are associated with the state of the local immune reaction. J Clin Oncol. 2011; 29:610-618.

18. Mizukami Y, Kono K, Kawaguchi Y, Akaike H, Kamimura K, Sugai H, Fujii H. Localisation pattern of Foxp3+ regulatory $\mathrm{T}$ cells is associated with clinical behaviour in gastric cancer. Br J Cancer. 2008; 98:148-153.

19. Curiel TJ. Tregs and rethinking cancer immunotherapy. J Clin Invest. 2007; 117:1167-1174.

20. Zou W. Regulatory $\mathrm{T}$ cells, tumour immunity and immunotherapy. Nat Rev Immunol. 2006; 6:295-307.

21. Dejaco C, Duftner C, Schirmer M. Analysis of FOXP3 protein expression in human $\mathrm{CD} 4(+) \mathrm{CD} 25(+)$ regulatory $\mathrm{T}$ cells at the single-cell level. Eur J Immunol. 2006; 36: 245-246; author reply 246.

22. Fontenot JD, Rasmussen JP, Williams LM, Dooley JL, Farr AG, Rudensky AY. Regulatory $\mathrm{T}$ cell lineage specification by the forkhead transcription factor foxp3. Immunity. 2005; 22:329-341.

23. Kono K, Kawaida H, Takahashi A, Sugai H, Mimura K, Miyagawa N, Omata H, Fujii H. CD4(+)CD25 high regulatory $\mathrm{T}$ cells increase with tumor stage in patients with gastric and esophageal cancers. Cancer Immunol Immunother. 2006; 55:1064-1071.

24. deLeeuw RJ, Kost SE, Kakal JA, Nelson BH. The prognostic value of FoxP3+ tumor-infiltrating lymphocytes in cancer: a critical review of the literature. Clin Cancer Res. 2012; 18:3022-3029.

25. Fontenot JD, Gavin MA, Rudensky AY. Foxp3 programs the development and function of CD4+CD25+ regulatory $\mathrm{T}$ cells. Nat Immunol. 2003; 4:330-336.

26. Hori S, Takahashi T, Sakaguchi S. Control of autoimmunity by naturally arising regulatory CD4+ T cells. Adv Immunol. 2003; 81:331-371.

27. Gooden MJ, de Bock GH, Leffers $\mathrm{N}$, Daemen $\mathrm{T}$, Nijman HW. The prognostic influence of tumour-infiltrating lymphocytes in cancer: a systematic review with metaanalysis. Br J Cancer. 2011; 105:93-103.

28. Haas M, Dimmler A, Hohenberger W, Grabenbauer GG, Niedobitek G, Distel LV. Stromal regulatory T-cells are associated with a favourable prognosis in gastric cancer of the cardia. BMC Gastroenterol. 2009; 9:65.

29. Ladoire S, Martin F, Ghiringhelli F. Prognostic role of FOXP3 + regulatory $\mathrm{T}$ cells infiltrating human carcinomas: the paradox of colorectal cancer. Cancer Immunol Immunother. 2011; 60:909-918.

30. Yoon HH, Orrock JM, Foster NR, Sargent DJ, Smyrk TC, Sinicrope FA. Prognostic impact of FoxP3+ regulatory T cells in relation to CD8+ T lymphocyte density in human colon carcinomas. PLoS One. 2012; 7:e42274. 
31. Lee HE, Park DJ, Kim WH, Kim HH, Lee HS. High FOXP3 + regulatory T-cell density in the sentinel lymph node is associated with downstream non-sentinel lymphnode metastasis in gastric cancer. Br J Cancer. 2011; 105: 413-419.

32. Perrone G, Ruffini PA, Catalano V, Spino C, Santini D, Muretto P, Spoto C, Zingaretti C, Sisti V, Alessandroni P, Giordani P, Cicetti A, D'Emidio S, et al. Intratumoural FOXP3-positive regulatory $\mathrm{T}$ cells are associated with adverse prognosis in radically resected gastric cancer. Eur J Cancer. 2008; 44:1875-1882.

33. Wang LH, Su L, Wang JT. Correlation between elevated FOXP3 expression and increased lymph node metastasis of gastric cancer. Chin Med J (Engl). 2010; 123:3545-3549.

34. Choi BD, Gedeon PC, Herndon JE, 2nd, Archer GE, Reap EA, Sanchez-Perez L, Mitchell DA, Bigner DD, Sampson JH. Human regulatory T cells kill tumor cells through granzyme-dependent cytotoxicity upon retargeting with a bispecific antibody. Cancer Immunol Res. 2013; 1:163.

35. Wang J, Ke XY. The four types of Tregs in malignant lymphomas. J Hematol Oncol. 2011; 4:50.

36. Sun HL, Zhou X, Xue YF, Wang K, Shen YF, Mao JJ, Guo HF and Miao ZN. Increased frequency and clinical significance of myeloid-derived suppressor cells in human colorectal carcinoma. World J Gastroenterol. 2012; 18:3303-3309.

37. Gabitass RF, Annels NE, Stocken DD, Pandha HA, Middleton GW. Elevated myeloid-derived suppressor cells in pancreatic, esophageal and gastric cancer are an independent prognostic factor and are associated with significant elevation of the Th2 cytokine interleukin-13. Cancer Immunol Immunother. 2011; 60:1419-1430.

38. Wang L, Chang EW, Wong SC, Ong SM, Chong DQ, Ling KL. Increased myeloid-derived suppressor cells in gastric cancer correlate with cancer stage and plasma S100A8/A9 proinflammatory proteins. J Immunol. 2013; 190:794-804.

39. Gabrilovich DI, Ostrand-Rosenberg S, Bronte V. Coordinated regulation of myeloid cells by tumours. Nat Rev Immunol. 2012; 12:253-268.
40. Nagaraj S, Schrum AG, Cho HI, Celis E, Gabrilovich DI. Mechanism of T cell tolerance induced by myeloid-derived suppressor cells. J Immunol. 2010; 184:3106-3116.

41. Ostrand-Rosenberg S. Myeloid-derived suppressor cells: more mechanisms for inhibiting antitumor immunity. Cancer Immunol Immunother. 2010; 59:1593-1600.

42. Ostrand-Rosenberg S, Sinha P. Myeloid-derived suppressor cells: linking inflammation and cancer. J Immunol. 2009; 182:4499-4506.

43. Sobin LH GM, Wittekind C. TNM classification of malignant tumors. 7th ed. New York: Oxford: WileyBlackwell; 2010.

44. Bu M, Shen Y, Seeger WL, An S, Qi R, Sanderson JA, Cai Y. Ovarian carcinoma-infiltrating regulatory $\mathrm{T}$ cells were more potent suppressors of CD8 T cell inflammation than their peripheral counterparts, a function dependent on TIM3 expression. Tumour Biol. 2015.

45. Chen MF, Kuan FC, Yen TC, Lu MS, Lin PY, Chung YH, Chen WC, Lee KD. IL-6-stimulated CD11b+ CD14+ HLADR- myeloid-derived suppressor cells, are associated with progression and poor prognosis in squamous cell carcinoma of the esophagus. Oncotarget. 2014; 5:8716-8728. doi: 10.18632/oncotarget.2368.

46. Chikamatsu K, Sakakura K, Toyoda M, Takahashi K, Yamamoto T, Masuyama K. Immunosuppressive activity of CD14+ HLA-DR - cells in squamous cell carcinoma of the head and neck. Cancer Sci. 2012; 103:976-983.

47. Fridman WH, Pages F, Sautes-Fridman C, Galon J. The immune contexture in human tumours: impact on clinical outcome. Nat Rev Cancer. 2012; 12:298-306.

48. Gutkin DW, Shurin MR. Clinical evaluation of systemic and local immune responses in cancer: time for integration. Cancer Immunol Immunother. 2014; 63:45-57.

49. Camp RL, Dolled-Filhart M, Rimm DL. X-tile: a new bioinformatics tool for biomarker assessment and outcomebased cut-point optimization. Clin Cancer Res. 2004; 10:7252-7259. 